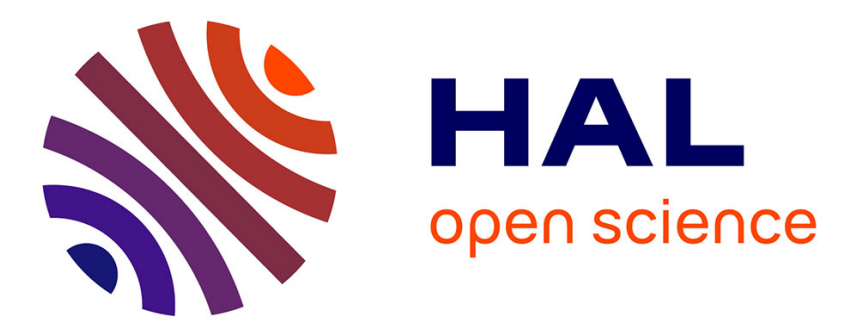

\title{
Complete shaking force and shaking moment balancing of planar parallel manipulators with prismatic pairs
}

\author{
Sébastien Briot, Ilian Bonev, Clément Gosselin, Vigen Arakelian
}

\section{To cite this version:}

Sébastien Briot, Ilian Bonev, Clément Gosselin, Vigen Arakelian. Complete shaking force and shaking moment balancing of planar parallel manipulators with prismatic pairs. Journal of Multi-body Dynamics, 2009, 223 (1), pp.43-52. hal-00451877

\section{HAL Id: hal-00451877 https://hal.science/hal-00451877}

Submitted on 25 Jun 2019

HAL is a multi-disciplinary open access archive for the deposit and dissemination of scientific research documents, whether they are published or not. The documents may come from teaching and research institutions in France or abroad, or from public or private research centers.
L'archive ouverte pluridisciplinaire HAL, est destinée au dépôt et à la diffusion de documents scientifiques de niveau recherche, publiés ou non, émanant des établissements d'enseignement et de recherche français ou étrangers, des laboratoires publics ou privés. 


\title{
Complete shaking force and shaking moment balancing of planar parallel manipulators with prismatic pairs
}

\author{
S. Briot", I.A. Bonev*, C.M. Gosselin ${ }^{\dagger}$ and V. Arakelian* \\ * Department of Automated Manufacturing Engineering \\ École de technologie supérieure (ÉTS), Montreal, QC, Canada \\ sebastien.briot@ens.etsmtl.ca \\ ilian.bonev@etsmtl.ca \\ ${ }^{\dagger}$ Département de génie mécanique \\ Université Laval, Sainte-Foy, QC, Canada \\ gosselin@gmc.ulaval.ca \\ \$Département de Génie Mécanique et Automatique \\ Institut National des Sciences Appliquées (INSA), Rennes, France \\ vigen.arakelyan@insa-rennes.fr
}

\begin{abstract}
This paper deals with the complete shaking force and shaking moment balancing of planar parallel manipulators with prismatic pairs. The cancellation of the dynamic loads transmitted to the ground is a challenge for these types of manipulators.
\end{abstract}


It is obvious that the classical methods based on the optimal redistribution of movable masses and additional counter-rotations can be used to cancel shaking force and shaking moment. However, such a balancing of parallel manipulators with prismatic pairs is only attained via a considerably complicated design. This paper shows that it is possible to balance planar parallel mechanisms using Scott-Russell mechanisms. Such an approach enables a division of the number of counter-rotations by two. Numerical simulations carried out using ADAMS software validate the obtained results and illustrate that the suggested balancing enables the creation of a parallel manipulator transmitting no inertia load to its base.

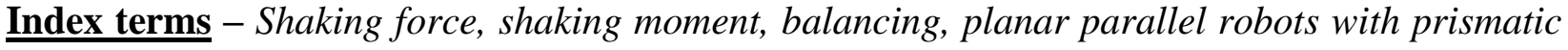
pairs.

\section{INTRODUCTION}

Shaking force balancing is mostly obtained via an optimal redistribution of movable masses [110] or adjustment of kinematic parameters [11]. The cancellation of the shaking moment is a more complicated task and can be obtained using three main different methods: (i) shaking moment balancing using counter-rotations [12-17] (Fig. 1a) (ii) shaking moment balancing by adding four bar linkages [18-22] (Fig.1b) and (iii) shaking moment balancing by optimal trajectory planning $[\mathbf{1 7}, \mathbf{2 3}, \mathbf{2 4}]$.

Previous works have been devoted to the study of parallel manipulators with revolute joints and until now, to our knowledge, no study has been carried out on complete shaking force and shaking moment balancing of parallel manipulators with prismatic pairs. 


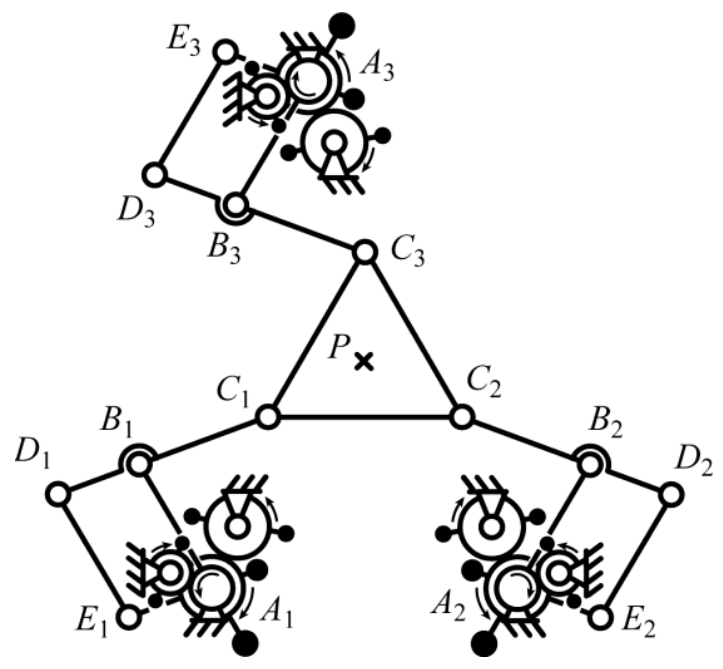

(a) balancing by adding counter-rotations [17]

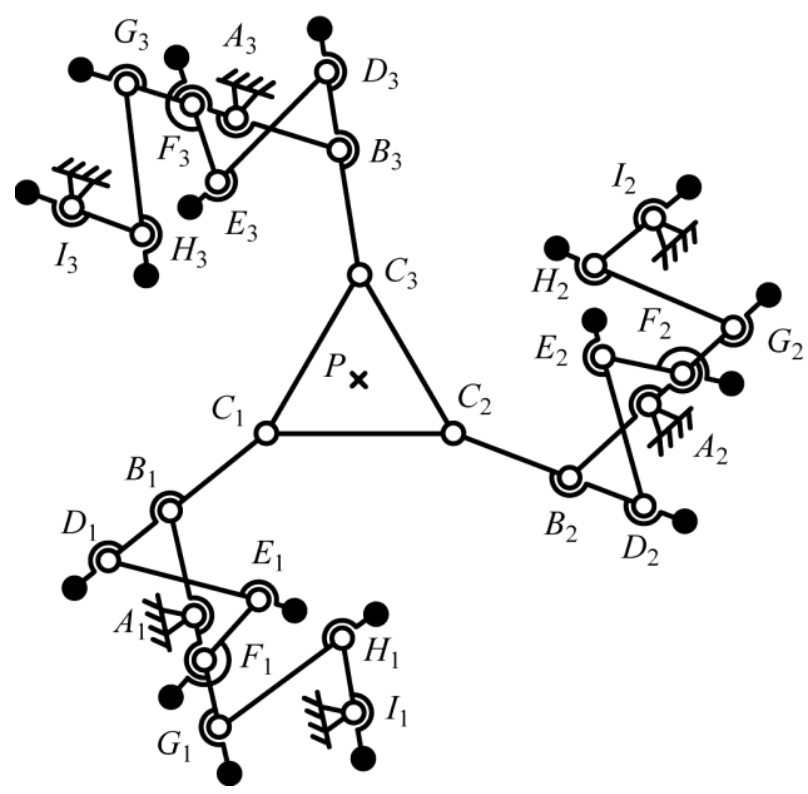

(b) balancing by adding four-bar linkages [18]

Figure 1. Complete shaking force and shaking moment balanced 3-RRR planar parallel manipulators.

In this paper, for the first time, we propose solutions for complete shaking force and shaking moment balancing of planar parallel manipulators with prismatic pairs. We illustrate these solutions via the 3-RPR parallel manipulator. All obtained results are validated using ADAMS software simulations.

\section{COMPLETE SHAKING MOMENT AND SHAKING FORCE BALANCING BY ADDING AN IDLER LOOP BETWEEN THE BASE AND THE PLATFORM}

Inertia force balancing by adding an idler loop is known to be used for 1-degree-of-freedom (DOF) mechanisms [25-29]. With regard to planar manipulators, such an approach has only been used in the balancing of gravitational and inertia forces $[9,10,30,31]$. 
In this section, the complete shaking force and shaking moment balancing of planar manipulators by adding an idler loop is discussed. The added balancing loop is mounted between the base and the platform of the mechanism. We illustrate the suggested balancing technique on a 3-RPR mechanism (Fig. 2). Please note that we do not mention the type of actuation of the mechanism as it has no influence on the balancing.

Firstly, let us analyze the cancellation of the dynamic reactions of the $3-R P R$ planar parallel mechanism (Fig. 2a). Such a mechanism has 3 DOF (two translations in the $\boldsymbol{O x y}$ plane and one rotation of the moving platform around an axis perpendicular to $\boldsymbol{O x y}$ ) and is composed of three identical legs, each being composed of a revolute joint attached to the base at point $A_{i}$ (in the remainder of this report, $i=1,2,3$ ), one moving prismatic guide, located at point $B_{i}$, and another revolute joint attached to the platform at $C_{i}$. The base and platform triangles, denoted $A_{1} A_{2} A_{3}$ and $C_{1} C_{2} C_{3}$, are equilateral. On this manipulator, typically, the actuated joints are the first revolute joint at $A_{i}$ or the linear guide at $B_{i}$.

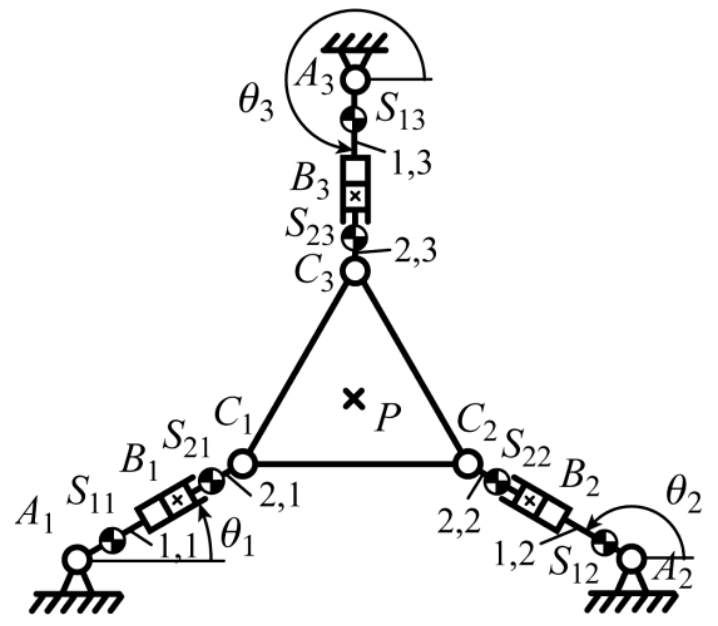

(a) Schematics of the 3-RPR mechanism.

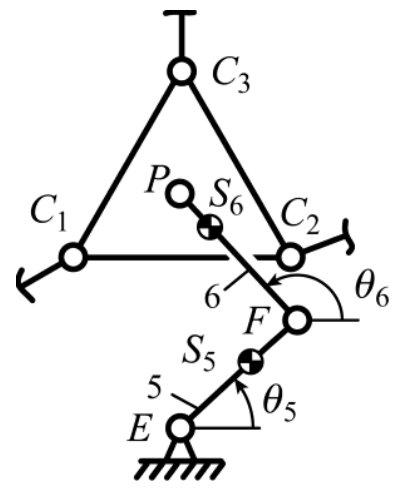

(b) Schematics of the added idler loop $(R R R$ chain).

Figure 2. Schematic of the 3-RPR robot under study. 
Considering that the $\boldsymbol{x}$ axis is directed along the line $A_{1} A_{2}$, the $\boldsymbol{y}$ axis being perpendicular to the $\boldsymbol{x}$ axis and the origin of the base frame located at point $O$, the centre of the circumcircle of triangle $A_{1} A_{2} A_{3}$, one can define the coordinate $x, y$ and $\phi$ of the platform, as being respectively the coordinates of point $P$ along the $\boldsymbol{x}$ and $\boldsymbol{y}$ axes and the angle between the line $C_{1} C_{2}$ and $A_{1} A_{2}$.

The length $B_{i} C_{i}$ is denoted $L_{1}$. Let us also denote as $S_{j i}$ the centre of mass of link $i j(j=1,2)$, which has a mass $m_{j}$ and an axial moment of inertia $I_{j}$. The centre of the mass of the platform is located at point $P$. The mass of the platform is $m_{p}$ and its axial moment of inertia $I_{p}$.

In order to cancel the shaking forces and shaking moment of the manipulator, an idler loop is added between the base and the platform (Fig. 2b). The lengths $E F$ and $F P$ are denoted $L_{5}$ and $L_{6}$ respectively. The centre of mass of elements 5 and 6 of the idler loop are denoted $S_{3}$ and $S_{4}$ with masses $m_{5}$ and $m_{6}$ and axial moments of inertia $I_{5}$ and $I_{6}$, respectively. The positions of the centre of masses are $\boldsymbol{d}_{A i S 1 i}=r_{1} L_{1} \boldsymbol{u}_{i}, \boldsymbol{d}_{C i S 2 i}=\left(r_{2}-1\right) L_{1} \boldsymbol{u}_{i}, \boldsymbol{d}_{E S 5}=r_{5} \boldsymbol{d}_{E F}$, and $\boldsymbol{d}_{F S 6}=r_{6} \boldsymbol{d}_{F P}, r_{1}, r_{2}, r_{5}$, and $r_{6}$ being dimensionless coefficients, and $\boldsymbol{u}_{i}$ a unit vector directed along $B_{i} C_{i}$.

Thus, considering the shaking force $\boldsymbol{F}$ of leg 1, its expression is:

$\boldsymbol{F}=\sum_{i=1}^{3} \sum_{j=1}^{2} m_{j} \ddot{\boldsymbol{d}}_{S j i}+m_{p} \ddot{\boldsymbol{d}}_{P}+m_{5} \ddot{\boldsymbol{d}}_{S 5}+m_{6} \ddot{\boldsymbol{d}}_{S 6}$

where $\ddot{\boldsymbol{d}}_{S j i}, \ddot{\boldsymbol{d}}_{P}, \ddot{\boldsymbol{d}}_{S 5}$ and $\ddot{\boldsymbol{d}}_{S 6}$ are the acceleration of the centre of mass $S_{i j}$, of $P$, of $S_{5}$ and of $S_{6}$ respectively.

After a simple development of expression (1), it can be seen that the shaking force $\boldsymbol{F}$ can be expressed as:

$\boldsymbol{F}=\left(m_{1} r_{1}-m_{2}\left(1-r_{2}\right)\right) \sum_{i=1}^{3} \boldsymbol{a}_{i}+\left(3 m_{2}+m_{p}+m_{6} r_{6}\right) \boldsymbol{a}_{4}+\left(3 m_{2}+m_{p}+m_{5} r_{5}+m_{6}\right) \ddot{\boldsymbol{d}}_{F}$

with 
$\boldsymbol{a}_{i}=L_{1}\left(\ddot{\theta}_{i}\left[\begin{array}{c}-\sin \theta_{i} \\ \cos \theta_{i}\end{array}\right]-\dot{\theta}_{i}^{2}\left[\begin{array}{c}\cos \theta_{i} \\ \sin \theta_{i}\end{array}\right]\right)$,

$\boldsymbol{a}_{4}=L_{6}\left(\ddot{\theta}_{6}\left[\begin{array}{c}-\sin \theta_{6} \\ \cos \theta_{6}\end{array}\right]-\dot{\theta}_{6}^{2}\left[\begin{array}{c}\cos \theta_{6} \\ \sin \theta_{6}\end{array}\right]\right)$,

and $\ddot{\boldsymbol{d}}_{F}$ is the acceleration of point $F$.

At this step, only five counterweights are needed in the cancellation of the shaking force, but it could be seen after more derivations that three others are necessary for the cancellation of the shaking moment. Therefore, we propose directly adding three supplementary counterweights (Fig. 3). The positions of the eight masses are $\boldsymbol{d}_{A i M c p 1 i}=r_{c p 1} L_{1} \boldsymbol{u}_{i}, \boldsymbol{d}_{B i M c p 2 i}=r_{c p 2} L_{1} \boldsymbol{u}_{i}, \boldsymbol{d}_{E M c p 5}=r_{c p 5}$ $\boldsymbol{d}_{E F}$, and $\boldsymbol{d}_{F M c p 6}=r_{c p} \boldsymbol{d}_{F P}, r_{c p 1}, r_{c p 2}, r_{c p 5}$, and $r_{c p 6}$ being dimensionless coefficients. Their masses are respectively denoted $m_{c p 1}, m_{c p 2}, m_{c p 5}$ and $m_{c p 6}$. With the addition of the counterweights, the shaking force becomes:

$\boldsymbol{F}^{b a l}=\boldsymbol{F}+\left(m_{c p 1} r_{c p 1}-m_{c p 2}\left(1-r_{c p 2}\right)\right) \sum_{i=1}^{3} \boldsymbol{a}_{i}+m_{c p 6} r_{c p 6} \boldsymbol{a}_{4}+\left(m_{c p 6}+m_{c p 5} r_{c p 5}\right) \ddot{\boldsymbol{d}}_{F}$

Thus, the shaking force is cancelled if:

$$
\begin{aligned}
& m_{c p 1}=-\frac{m_{1} r_{1}}{r_{c p 1}}, \\
& m_{c p 2}=-\frac{m_{2}\left(1-r_{2}\right)}{1-r_{c p 2}}, \\
& m_{c p 6}=-\frac{3\left(m_{2}+m_{c p 2}\right)+m_{p}+r_{6} m_{6}}{r_{c p 6}},
\end{aligned}
$$

and 
$m_{c p 5}=-\frac{3\left(m_{2}+m_{c p 2}\right)+m_{p}+m_{6}+m_{c p 6}+r_{5} m_{5}}{r_{c p 5}}$

The expression of the shaking moment $M_{O}$ of the modified structure (expressed at point $O$ ) can be written as:

$M_{O}=\frac{d H_{O}}{d t}$

where $H_{O}$ is the angular momentum of the leg (expressed at point $O$ ). Thus, in order to cancel the shaking moment, the angular momentum is held constant over time.

The expression of the angular momentum $H_{O}$ is:

$$
\begin{aligned}
H_{O}= & \sum_{i=1}^{3} \sum_{j=1}^{2}\left(m_{j}\left(x_{O S j i} \dot{y}_{O S j i}-y_{O S j i} \dot{x}_{O S j i}\right)+I_{j} \dot{\theta}_{i}\right)+\sum_{i=1}^{3} \sum_{j=1}^{2}\left(m_{c p j}\left(x_{O M j i} \dot{y}_{O M j i}-y_{O M j i} \dot{x}_{O M j i}\right)\right)+I_{p} \dot{\phi} \\
& +\sum_{j=5}^{6}\left(m_{j}\left(x_{O S j} \dot{y}_{O S j}-y_{O S j} \dot{x}_{O S j}\right)+m_{c p j}\left(x_{O M j} \dot{y}_{O M j}-y_{O M j} \dot{x}_{O M j}\right)+I_{j} \dot{\theta}_{j}\right)
\end{aligned}
$$

where $x_{O Q}, y_{O Q}, \dot{x}_{O Q}$ and $\dot{y}_{O Q}$ are the position and velocities of any point $Q$ along $\boldsymbol{x}$ and $\boldsymbol{y}$ axes, respectively, $Q$ being either point $S_{j i}, M_{j i},(j=1,2), S_{j}$ or $M_{j}(j=5,6)$.

Developing and introducing (5) into (7) yields

$$
\begin{aligned}
H_{O}= & \sum_{i=1}^{3}\left(I_{1}+I_{2}+\left(m_{1} r_{1}^{2}+m_{c p 1} r_{c p 1}^{2}+m_{2}\left(1-r_{2}\right)^{2}\right) L_{1}^{2}\right) \dot{\theta}_{i}+\sum_{i=1}^{3}\left(m_{c p 2}\left(1-r_{c p 2}\right)^{2} L_{1}^{2}\right) \dot{\theta}_{i} \\
& +\left(I_{p}+3\left(m_{2}+m_{c p 2}\right) R_{p}^{2}\right) \dot{\phi}+\left(I_{6}+\left(m_{6} r_{6}^{2}+m_{c p 6} r_{c p 6}^{2}+m_{p}+3\left(m_{2}+m_{c p 2}\right)\right) L_{6}^{2}\right) \dot{\theta}_{6} \\
& +\left(I_{5}+\left(m_{5} r_{5}^{2}+m_{c p 5} r_{c p 5}^{2}+m_{6}+m_{c p 6}+m_{p}\right) L_{5}^{2}\right) \dot{\theta}_{5}+3\left(m_{2}+m_{c p 2}\right) L_{5}^{2} \dot{\theta}_{5}
\end{aligned}
$$

After such modifications of the $R R R$ chain, the angular momentum of the legs of the mechanism and of the $R R R$ chain can be balanced using six counter-rotations (Fig. 3), which have an axial moment of inertia equal to: 


$$
\begin{aligned}
& I_{c r 1}=I_{1}+I_{2}+\left(m_{1} r_{1}^{2}+m_{c p 1} r_{c p 1}^{2}+m_{2}\left(1-r_{2}\right)^{2}+m_{c p 2}\left(1-r_{c p 2}\right)^{2}\right) L_{1}^{2} \\
& I_{c r 2}=I_{p}+3\left(m_{2}+m_{c p 2}\right) R_{p}^{2} \\
& I_{c r 3}=\left(m_{6} r_{6}^{2}+m_{c p 6} r_{c p 6}^{2}+m_{p}+3\left(m_{2}+m_{c p 2}\right)\right) L_{6}^{2}+2 I_{c r 2}+I_{6} \\
& I_{c r 4}=\left(m_{5} r_{5}^{2}+m_{c p 5} r_{c p 5}^{2}+m_{6}+m_{c p 6}+m_{p}+3\left(m_{2}+m_{c p 2}\right)\right) L_{5}^{2}+2 I_{c r 3}+I_{5}
\end{aligned}
$$

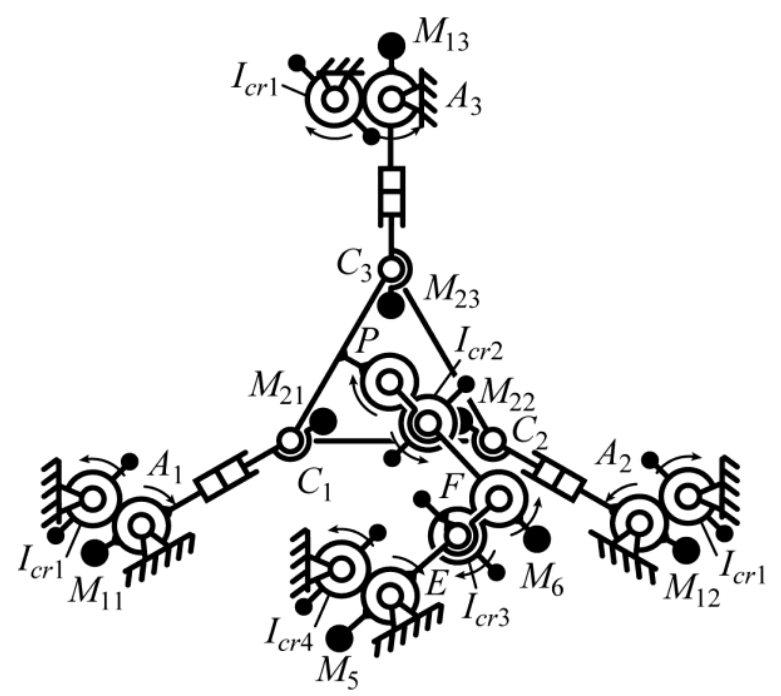

Figure 3. Schematics of the 3-RPR mechanism with the added $R R R$ chain used for the cancellation of the shaking force and shaking moment.

\section{Numerical application}

Let us illustrate the suggested balancing approach using numerical simulations carried out with ADAMS software. For this purpose, non balanced and balanced 3-RPR parallel manipulators will be compared.

The chosen trajectory for simulations is a straight line of the controlled point of the platform, achieved in $t_{f}=0.25 \mathrm{~s}$, between $P_{0}=\left(x_{0}, y_{0}\right)=(-0.05 \mathrm{~m}, 0)$ and $P_{f}=\left(x_{f}, y_{f}\right)=(-0.2 \mathrm{~m}, 0)$ with a 
rotation of the platform from $\phi_{0}=0^{\circ}$ to $\phi_{f}=30^{\circ}$. For the displacement of the mechanism, fifth order polynomial laws are used and therefore the trajectory is defined by the following expressions:

$x(t)=x_{0}+\left(x_{f}-x_{0}\right)\left(10\left(t / t_{f}\right)^{3}-15\left(t / t_{f}\right)^{4}+6\left(t / t_{f}\right)^{5}\right)$,

$y(t)=0$

$\phi(t)=\phi_{0}+\left(\phi_{f}-\phi_{0}\right)\left(10\left(t / t_{f}\right)^{3}-15\left(t / t_{f}\right)^{4}+6\left(t / t_{f}\right)^{5}\right)$.

The parameters used for the simulations are the following:

- $\quad$ radii of the circumcircles of the base triangle $A_{1} A_{2} A_{3}$ and the platform triangle $C_{1} C_{2} C_{3}, R_{b}$ $=0.35 \mathrm{~m}, R_{p}=0.1 \mathrm{~m}$;

- $\quad L_{1}=0.05 \mathrm{~m} ; L_{5}=0.15 \mathrm{~m} ; L_{6}=0.1581 \mathrm{~m} ;$

$-r_{2}=r_{5}=r_{6}=0.5 ; r_{1}=2$;

- $\quad m_{1}=0.75 \mathrm{~kg} ; m_{2}=0.37 \mathrm{~kg} ; m_{5}=0.42 \mathrm{~kg} ; m_{6}=0.47 \mathrm{~kg} ; m_{p}=1 \mathrm{~kg}$

- $\quad I_{1}=0.00344 \mathrm{~kg} \cdot \mathrm{m}^{2} ; I_{2}=0.00025 \mathrm{~kg} \cdot \mathrm{m}^{2} ; I_{5}=0.00122 \mathrm{~kg} \cdot \mathrm{m}^{2} ; I_{6}=0.00146 \mathrm{~kg} \cdot \mathrm{m}^{2} ; I_{p}=$ 0.00436 kg.m;

- $\quad$ point $E$ is located at point $O$.

For such parameters and such a trajectory, the shaking force and shaking moment are computed using ADAMS software and are presented in Fig. 4 (solid line). Then, we add the counterweights and the idler loop EFP to the mechanism. The position coefficients of the counterweights are all equal to $r_{c p j}=0.5(j=1,2,5,6)$. Therefore, the added masses are equal to $m_{c p 1}=0.75 \mathrm{~kg}, m_{c p 2}=$ $0.37 \mathrm{~kg}, m_{c p 5}=6.92 \mathrm{~kg}, m_{c p 6}=21.66 \mathrm{~kg}$. The new values of the shaking force and moment are presented in Fig. 4 (dashed line). It is possible to see that with the added counterweights the 
shaking efforts are cancelled, while the maximal value of shaking moment is increased by a factor 17. Finally, we add the counter-rotations. Their values are equal to $I_{c r 1}=0.01917 \mathrm{~kg} \cdot \mathrm{m}^{2}$, $I_{c r 2}=0.02665 \mathrm{~kg} \cdot \mathrm{m}^{2}, I_{c r 3}=0.18169 \mathrm{~kg} \cdot \mathrm{m}^{2}, I_{c r 4}=0.72781 \mathrm{~kg} . \mathrm{m}^{2}$. With such counter-rotations, the shaking moment is balanced (in gray line in Fig. 4c).



(a) shaking force along $\boldsymbol{x}$-axis

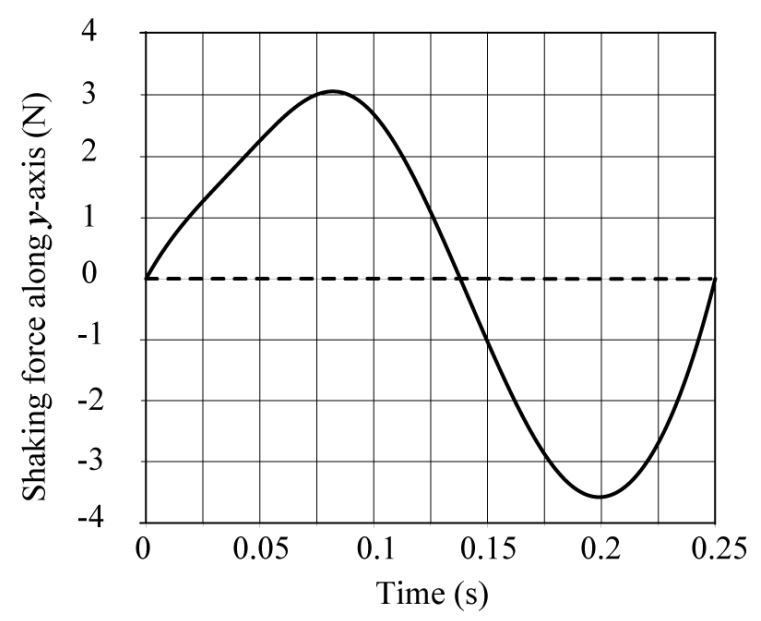

(b) shaking force along $\boldsymbol{y}$-axis

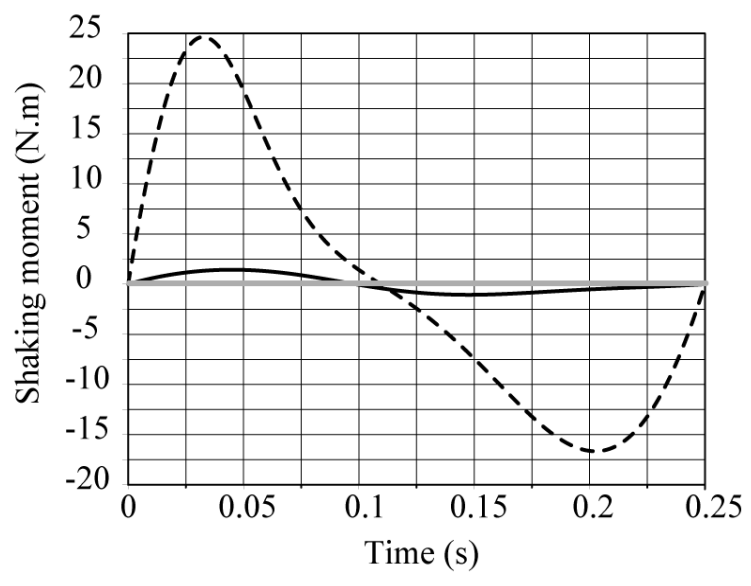

(c) shaking moment along $z$-axis

Figure 4. Shaking force and shaking moment before (solid line) and after (dashed line) the addition of the counterweights, and after the addition of the counter-rotations (gray line). 


\section{COMPLETE SHAKING FORCE AND SHAKING MOMENT BALANCING VIA SCOTT-RUSSELL MECHANISM}

In this section another approach for complete shaking force and shaking moment balancing is developed, which consists of adding Scott-Russell mechanisms to the initial architecture of a manipulator. This approach enables a reduction in the number of counter-rotations.

\subsection{Properties of the Scott-Russell mechanism.}

Firstly, let us observe a simple slider-crank mechanism (Fig. 5). Let us denote the lengths $A B$ and $B C$ as respectively $L_{1}$ and $L_{2}$, and the centre of masses of link $i(i=1,2,3)$ as $S_{i}$, which has a mass $m_{i}$ and an axial moment of inertia $I_{i}$. The positions of the centres of mass are $\boldsymbol{d}_{A S 1}=r_{1} \boldsymbol{d}_{A B}$, $\boldsymbol{d}_{A S 2}=\boldsymbol{d}_{A B}+r_{2} \boldsymbol{d}_{B C}, \boldsymbol{d}_{A S 3}=\boldsymbol{d}_{A C}+\boldsymbol{d}_{C S 3}, r_{1}$ and $r_{2}$ being dimensionless coefficients, $\boldsymbol{d}_{C S 3}=L_{3} r_{3} \boldsymbol{x}$, ( $L_{3}$ is a constant).



Figure 5. A general slider-crank mechanism.

It is known that the compete shaking force and shaking moment balancing of a general slidercrank mechanism can be obtained by adding two counterweights mounted on the links and two pairs counter-rotation inertia-counterweights. However, it is possible to balance this mechanism 
without counter-rotation inertia-counterweights if it has specific geometrical parameters, as in Scott-Russell mechanisms $\left(a=0, L_{1}=L_{2}\right)$.

Let us consider the balancing of this mechanism. The expression of the shaking force $\boldsymbol{F}$ of a slider-crank mechanism can be written as:

$\boldsymbol{F}=\sum_{i=1}^{3} m_{i} \ddot{\boldsymbol{d}}_{S i}$

where $\ddot{\boldsymbol{d}}_{S i}$ is the acceleration of the centre of mass $S_{i}$.

Developing (11), the expression of $\boldsymbol{F}$ becomes:

$\boldsymbol{F}=\left(m_{1} r_{1}+m_{2}\right) \ddot{\boldsymbol{d}}_{B}+\left(m_{2} r_{2}+m_{3}\right) \boldsymbol{a}$

with

$\boldsymbol{a}=L_{2}\left(\ddot{\theta}_{2}\left[\begin{array}{c}-\sin \theta_{2} \\ \cos \theta_{2}\end{array}\right]-\dot{\theta}_{2}^{2}\left[\begin{array}{c}\cos \theta_{2} \\ \sin \theta_{2}\end{array}\right]\right)$.

$\ddot{d}_{B}$ is the acceleration of point $B$.

The constant terms of eq. (12) can be cancelled by the addition of two counterweights $M_{j}$, $(j=$ 1, 2) (Fig. 5), of which the masses are $m_{c p j}$. Their positions are: $\boldsymbol{d}_{A M c p 1}=r_{c p 1} \boldsymbol{d}_{A B}, \boldsymbol{d}_{B M c p 2}=r_{c p 2} \boldsymbol{d}_{B C}$, $r_{c p 1}$ and $r_{c p 2}$ being dimensionless coefficients. With the addition of the counterweights, the shaking force becomes:

$\boldsymbol{F}^{b a l}=\boldsymbol{F}+\left(m_{c p 1} r_{c p 1}+m_{c p 2}\right) \ddot{\boldsymbol{d}}_{B}+m_{c p 2} r_{c p 2} \boldsymbol{a}$

Thus, the shaking force vanishes if:

$m_{c p 2}=-\frac{m_{2} r_{2}+m_{3}}{r_{c p 2}}$ and $m_{c p 1}=-\frac{m_{1} r_{1}+m_{2}+m_{c p 2}+m_{3}}{r_{c p 1}}$ 
The expression of the angular momentum $H_{A}$ (expressed at point $A$ ) is:

$H_{A}=\sum_{j=1}^{3}\left(m_{j}\left(x_{A S j} \dot{y}_{A S j}-\dot{x}_{A S j} y_{A S j}\right)\right)+\sum_{j=1}^{2}\left(I_{j} \dot{\theta}_{j}+m_{c p j}\left(x_{M S j} \dot{y}_{M S j}-\dot{x}_{M S j} y_{M S j}\right)\right)$

where $x_{A Q}, y_{A Q}, \dot{x}_{A Q}$ and $\dot{y}_{A Q}$ are the position and velocities of any point $Q$ along $\boldsymbol{x}$ and $\boldsymbol{y}$ axes, respectively, $Q$ being either point $S_{j}$ or $M_{j}(j=1,2,3)$.

Developing and introducing (15) into (16),

$H_{A}=\left(I_{1}+\left(m_{1} r_{1}^{2}+m_{c p 1} r_{c p 1}^{2}+m_{2}+m_{c p 2}+m_{3}\right) L_{1}^{2}\right) \dot{\theta}_{1}+\left(I_{2}+\left(m_{2} r_{2}^{2}+m_{c p 2} r_{c p 2}^{2}+m_{3}\right) L_{2}^{2}\right) \dot{\theta}_{2}$

with

$\dot{\theta}_{2}=-\frac{\dot{y}_{A B}\left(x_{A C}-x_{A B}\right)+\left(a-y_{A B}\right)\left(\dot{x}_{A C}-\dot{x}_{A B}\right)}{L_{2}^{2}}$.

where $x_{A C}, x_{A B}, y_{A B}$ are the coordinates of points $C$ and $B$, respectively, and $\dot{x}_{A C}, \dot{x}_{A B}, \dot{y}_{A B}$ their velocities.

In order to cancel the shaking moment $M_{A}$, the angular momentum has to be constant or null. Developing (18), one notices that this can be obtained if:

$a=0$ and $L_{1}=L_{2}$.

In such a case, $\dot{\theta}_{1}=-\dot{\theta}_{2}$. Therefore, the shaking moment is cancelled if:

$I_{1}+\left(m_{1} r_{1}^{2}+m_{c p 1} r_{c p 1}^{2}+m_{2}+m_{c p 2}\right) L_{1}^{2}-I_{2}-\left(m_{2} r_{2}^{2}+m_{c p 2} r_{c p 2}^{2}\right) L_{1}^{2}=0$ 


\subsection{Balancing of a manipulator's leg using a Scott-Russell mechanism.}

Now let us consider a manipulator's leg with an added Scott-Russell mechanism (Fig. 6). Let us denote as $S_{4}$ the centre of mass of link 4 , which has a mass $m_{4}$ and an axial moment of inertia $I_{4}$. The position of $S_{4}$ is: $\mathbf{d}_{C S 4}=L_{3} r_{4} \mathbf{u}, r_{4}$ being a dimensionless coefficient and $\mathbf{u}$ a unit vector along $\mathbf{d}_{C S 3}$.

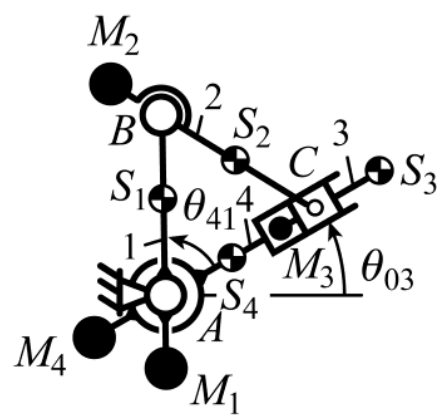

Figure 6. A manipulator leg with added Scott-Russell mechanism.

Now the shaking force becomes:

$\boldsymbol{F}=\left(m_{1} r_{1}+m_{c p 1} r_{c p 1}+m_{2}+m_{c p 2}+m_{3}\right) \ddot{\boldsymbol{d}}_{B}+\left(m_{3} r_{3}+m_{4} r_{4}\right) \boldsymbol{a}_{1}+\left(m_{2} r_{2}+m_{c p 2} r_{c p 2}+m_{3}\right) \boldsymbol{a}_{2}$

with

$\boldsymbol{a}_{1}=L_{3}\left(\ddot{\theta}_{03}\left[\begin{array}{c}-\sin \theta_{03} \\ \cos \theta_{03}\end{array}\right]-\dot{\theta}_{03}^{2}\left[\begin{array}{c}\cos \theta_{03} \\ \sin \theta_{03}\end{array}\right]\right)$

and

$\boldsymbol{a}_{2}=L_{1}\left(\left(\ddot{\theta}_{03}-\ddot{\theta}_{41}\right)\left[\begin{array}{c}-\sin \left(\theta_{03}-\theta_{41}\right) \\ \cos \left(\theta_{03}-\theta_{41}\right)\end{array}\right]-\left(\dot{\theta}_{03}-\dot{\theta}_{03}\right)^{2}\left[\begin{array}{c}\cos \left(\theta_{03}-\theta_{41}\right) \\ \sin \left(\theta_{03}-\theta_{41}\right)\end{array}\right]\right)$.

At this step, only one counterweight is needed for the cancellation of the shaking force, but it could be seen after more derivations that another is necessary for the cancellation of the shaking moment. Therefore, we propose adding this supplementary counterweight directly. The positions 
of the two masses are: $\boldsymbol{d}_{A M c p 3}=r_{c p 3} L_{3} \boldsymbol{u}, \boldsymbol{d}_{C M c p 4}=r_{c p 4} L_{3} \boldsymbol{u}, r_{c p 3}$ and $r_{c p 4}$ being dimensionless coefficients. Their masses are respectively denoted $m_{c p 3}$ and $m_{c p 4}$. With the addition of the counterweights, the shaking force becomes:

$\boldsymbol{F}^{b a l}=\boldsymbol{F}+m_{c p 3} \ddot{\boldsymbol{d}}_{B}+m_{c p 3} \boldsymbol{a}_{1}+\left(m_{c p 3} r_{c p 3}+m_{c p 4} r_{c p 4}\right) \boldsymbol{a}_{2}$.

Thus, the shaking force is cancelled if:

$m_{c p 4}=-\frac{m_{4} r_{4}}{r_{c p 4}}$

$m_{c p 3}=-\frac{m_{3} r_{3}}{r_{c p 3}}$

$m_{c p 2}=-\frac{m_{2} r_{2}+m_{3}+m_{c p 3}}{r_{c p 2}}$

and $m_{c p 1}=-\frac{m_{1} r_{1}+m_{2}+m_{c p 2}+m_{3}+m_{c p 3}}{r_{c p 1}}$

Developing and simplifying, the expression of the angular momentum is:

$H_{A}=I_{e q 1} \dot{\theta}_{03}+I_{e q 2} \dot{\theta}_{41}$

with

$$
\begin{aligned}
I_{e q 1}= & \left(m_{1} r_{1}^{2}+m_{c p 1} r_{c p 1}^{2}+m_{2}\left(1-r_{2}\right)^{2}+m_{c p 2}\left(1-r_{c p 2}\right)^{2}\right) L_{1}^{2}+I_{1}+I_{2}+I_{3}+I_{4} \\
& +\left(m_{3} r_{3}^{2}+m_{c p 3} r_{c p 3}^{2}+m_{4} r_{4}^{2}+m_{c p 4} r_{c p 4}^{2}\right) L_{3}^{2} \\
I_{e q 2}= & I_{1}+\left(m_{1} r_{1}^{2}+m_{c p 1} r_{c p 1}^{2}+m_{2}+m_{c p 2}\right) L_{1}^{2}-I_{2}-\left(m_{2} r_{2}^{2}+m_{c p 2} r_{c p 2}^{2}\right) L_{1}^{2} .
\end{aligned}
$$


From (20), $I_{e q 2}=0$. Therefore, the shaking moment of the slider-crank can be cancelled using a simple counter-rotation $I_{c r}$ with an axial moment of inertia equal to $I_{e q 1}$.

\subsection{Shaking moment and shaking force balancing of the 3-RPR manipulator}

Now, let us apply such an approach to the 3-RPR mechanism. First of all, let us substitute the platform mass by three points masses located at $C_{1}, C_{2}$ and $C_{3}$, with the values of mass equal to $m_{p 1}, m_{p 2}$ and $m_{p 3}$ respectively $[\mathbf{1 3}, \mathbf{3 1}, \mathbf{3 2}]$. Such a condition can be obtained if:

$m_{p i}=m_{p} / 3$ and $I_{p}=3 m_{p i} R_{p}^{2}$.

where $R_{p}$ is the radius of the circumcircle of $C_{1} C_{2} C_{3}$. Such a decomposition of the platform enables us to consider the shaking force and shaking moment balancing of each leg of the mechanism. Then, modifying each leg in order to obtain a mechanism similar to a slider-crank linkage, (i.e., by adding an idler loop to each leg), the shaking force and shaking moment are cancelled if:

$$
\begin{aligned}
& m_{c p 4}=-\frac{m_{4} r_{4}}{r_{c p 4}}, \\
& m_{c p 3}=-\frac{m_{3} r_{3}+m_{p i}}{r_{c p 3}}, \\
& m_{c p 2}=-\frac{m_{2} r_{2}+m_{3}+m_{c p 3}+m_{p i}}{r_{c p 2}}, \\
& m_{c p 1}=-\frac{m_{1} r_{1}+m_{2}+m_{c p 2}+m_{3}+m_{c p 3}+m_{p i}}{r_{c p 1}}, \\
& 0=I_{1}+\left(m_{1} r_{1}^{2}+m_{c p 1} r_{c p 1}^{2}+m_{2}+m_{c p 2}\right) L_{1}^{2}-I_{2}-\left(m_{2} r_{2}^{2}+m_{c p 2} r_{c p 2}^{2}\right) L_{1}^{2},
\end{aligned}
$$


and

$$
\begin{aligned}
I_{c r}= & \left(m_{1} r_{1}^{2}+m_{c p 1} r_{c p 1}^{2}+m_{2}\left(1-r_{2}\right)^{2}+m_{c p 2}\left(1-r_{c p 2}\right)^{2}\right) L_{1}^{2}+I_{1}+I_{2} \\
& +I_{3}+I_{4}+\left(m_{3} r_{3}^{2}+m_{c p 3} r_{c p 3}^{2}+m_{4} r_{4}^{2}+m_{c p 4} r_{c p 4}^{2}+m_{p i}\right) L_{3}^{2}
\end{aligned}
$$

taking into account that $I_{c r}$ is the axial moment of inertia of the counter-rotations (Fig. 7).

Thus, with this approach it is possible to create a fully-balanced shaking force and shaking moment 3-RPR mechanism with only three counter-rotations (Fig. 7), i.e., this method enables a reduction in the number of counter-rotations by a factor of two.

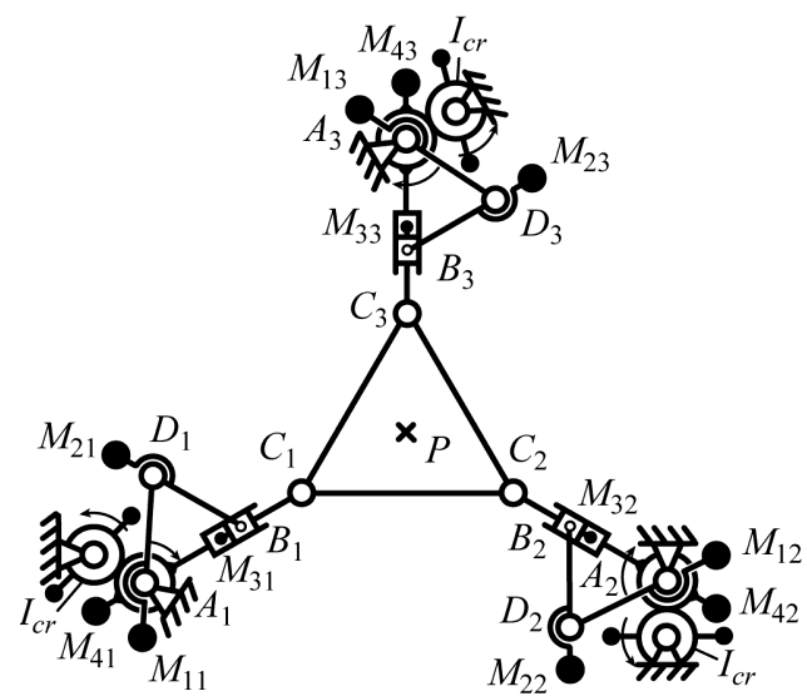

Figure 7. Schematics of a shaking force and shaking moment balanced $3-R P R$ mechanism.

\subsection{Numerical application}

The parameters used for the simulations are the followings:

- $\quad$ radii of the circumcircles of the base triangle $A_{1} A_{2} A_{3}$ and the platform triangle $C_{1} C_{2} C_{3} R_{b}$ $=0.35 \mathrm{~m}, R_{p}=0.1 \mathrm{~m} ;$

- $\quad L_{1}=L_{2}=0.25 \mathrm{~m} ; L_{3}=0.025 \mathrm{~m}$ 
$-\quad r_{1}=r_{2}=0.5 ; r_{3}=0 ; r_{4}=4$

- $\quad m_{1}=1.09 \mathrm{~kg} ; m_{2}=1.1 \mathrm{~kg} ; m_{3}=0.37 \mathrm{~kg} ; m_{4}=0.75 \mathrm{~kg} ; m_{p}=1 \mathrm{~kg}$

$-\quad I_{1}=0.00738 \mathrm{~kg} \cdot \mathrm{m}^{2} ; I_{2}=0.58389 \mathrm{~kg} \cdot \mathrm{m}^{2} ; I_{3}=0.00344 \mathrm{~kg} \cdot \mathrm{m}^{2} ; I_{6}=0.00025 \mathrm{~kg} \cdot \mathrm{m}^{2} ; I_{p}=0.01$ kg.m $\mathrm{m}^{2}$

For these new parameters and for the trajectory used previously, taking into account that the position coefficients of the counterweights are equal to $r_{c p j}=-0.5(j=1,3,4), r_{c p 2}=-1$, the new values of the counterweights are: $m_{c p 1}=3.17 \mathrm{~kg}, m_{c p 2}=11.71 \mathrm{~kg}, m_{c p 3}=0.33 \mathrm{~kg}, m_{c p 4}=0.75 \mathrm{~kg}$. The shaking force and shaking moment are then computed (dashed line in Fig. 8). It is possible to see that, with the counterweights, the shaking efforts are cancelled, while the maximal value of the shaking moment is increased by a factor 28 . Finally, we add the counter-rotations. Their values are equal to $I_{c r}=1.56907 \mathrm{~kg} \cdot \mathrm{m}^{2}$. With such counter-rotations, the shaking moment is balanced (gray line in Fig. 8c).

Finally, it should be noted that the combination of the proposed two techniques of balancing enables the creation of fully balanced parallel manipulators with modified legs. As examples, different structures of balanced manipulators are presented in Fig. 9 (3-RPR, 3-PRR and 3-PRP) in which one leg with a prismatic pair is replaced by a leg with only revolute joints. Such a modification allows displacing the centre of mass of the manipulator to $C_{3}$ and then to balance the manipulator via the modified leg $C_{3} B_{3} A_{3}$.

In the same way, it is possible to balance a parallel manipulator with prismatic pairs by adding fewer Scott-Russell mechanisms. The balancing schemes for several parallel manipulators are presented in Fig. 10. 




(a) shaking force along $\boldsymbol{x}$-axis



(b) shaking force along $\boldsymbol{y}$-axis

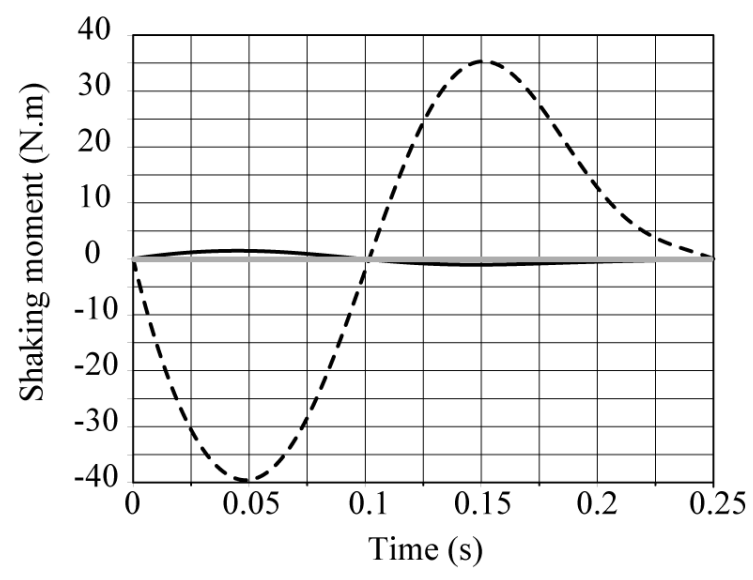

(c) shaking moment along $z$-axis

Figure 8. Shaking force and shaking moment, before (solid line) and after (dashed line) the addition of the counterweights, and after the addition of the counter-rotations (gray line). 




a) Modified 3-RPR parallel manipulator

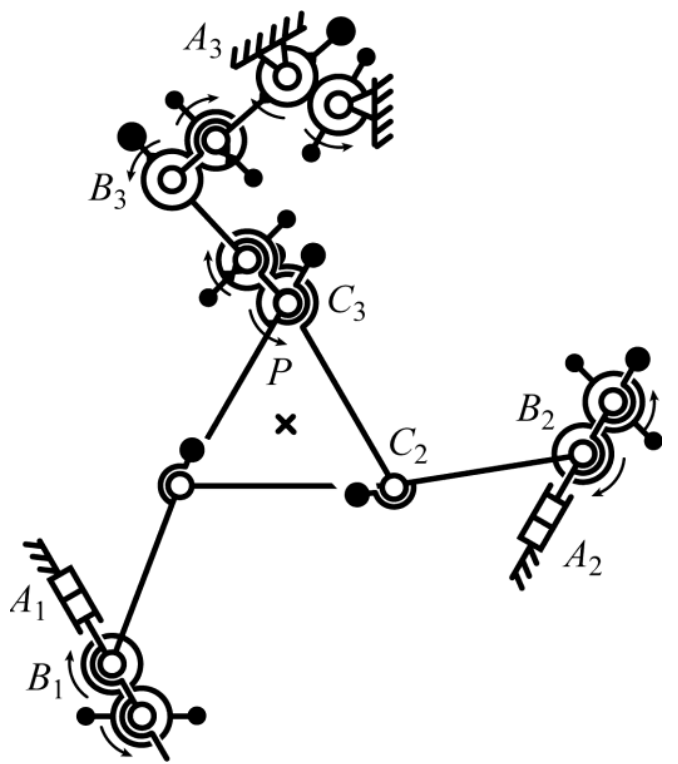

b) Modified 3-PRR parallel manipulator

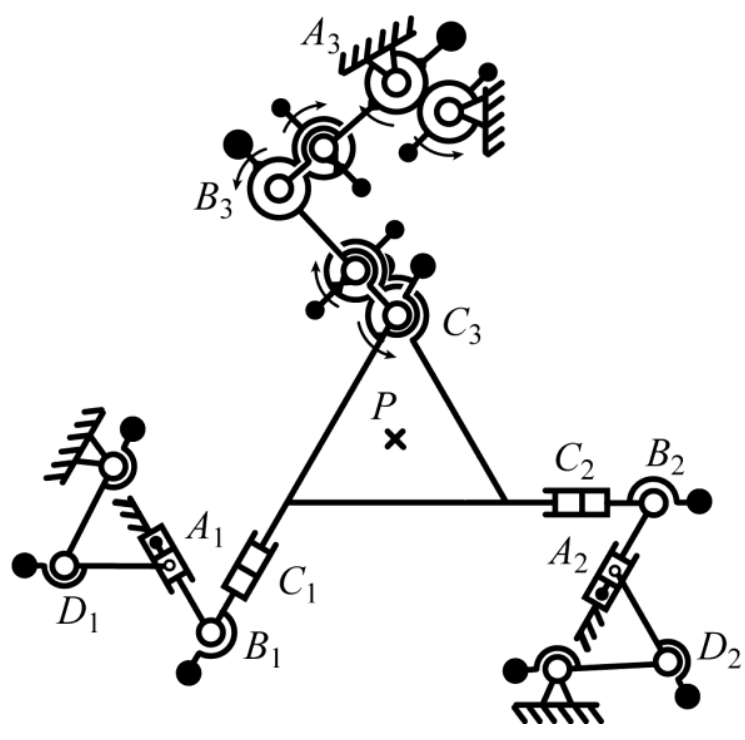

c) Modified 3-PRP parallel manipulator

Figure 9. Complete shaking force and shaking moment balancing of planar manipulators with prismatic pairs via structural modification of one leg. 


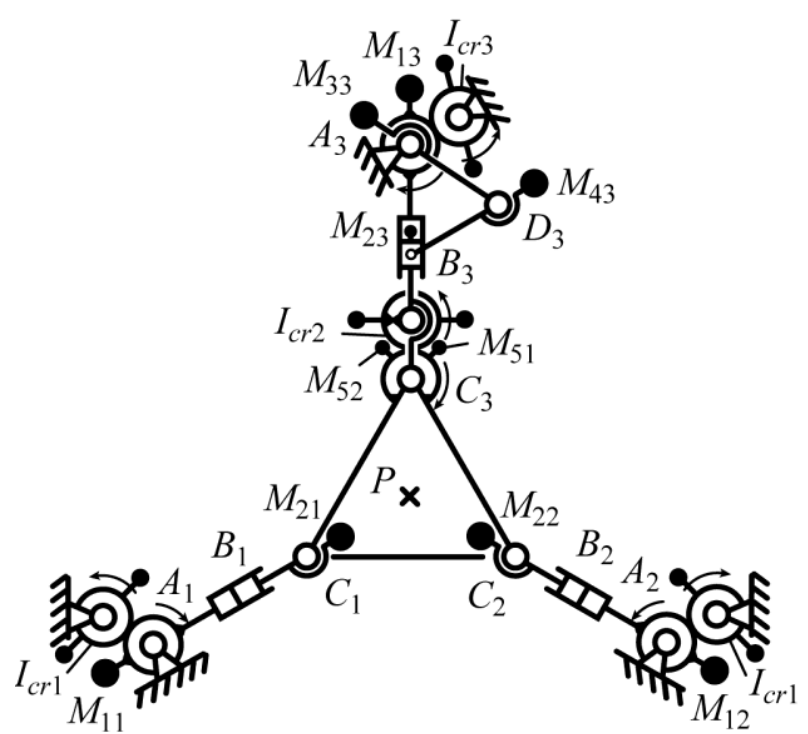

a) Balancing of 3-RPR parallel manipulator

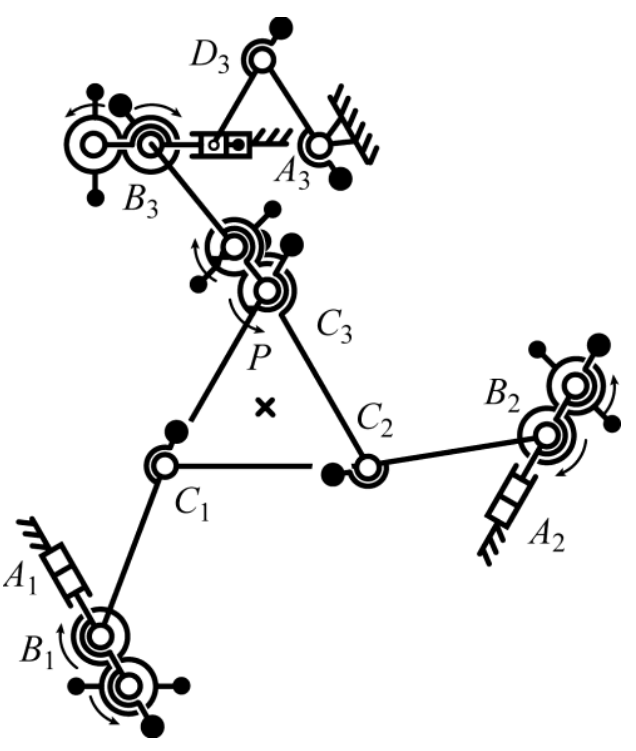

b) Balancing of 3-PRR parallel manipulator

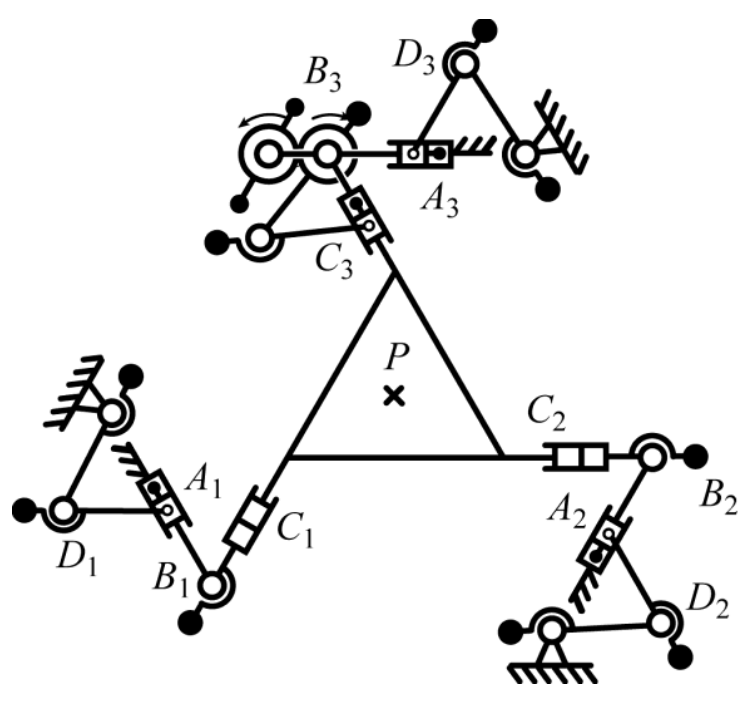

c) Balancing of 3-PRP parallel manipulator

Figure 10. Complete shaking force and shaking moment balancing of planar manipulators with prismatic with reduced number of Scott-Russell mechanisms. 


\section{CONCLUSIONS}

This paper presents the complete shaking force and shaking moment balancing of planar parallel manipulators with prismatic pairs. Two approaches are discussed: balancing via adding an idler loop mounted between the platform and the base of the manipulator and balancing via the Scott-Russell mechanism, which enables a reduction in the number of counter-rotations by a factor of two. All studied balancing techniques are validated by simulations carried out using ADAMS software. The obtained results show that parallel manipulators balanced using the suggested methods transmit no inertia loads to their bases, i.e., the sum of all ground forces and their moments are zero.

Finally, we would like to mention that using Scott-Russel mechanisms remains using some modified 3-RRR manipulator. Thus, if we do not consider the type of actuation, the prismatic guides could be suppressed and our work could be of no interest. But the goal of our study is to propose the complete shaking force and shaking moment balancing of manipulators for applications where an actuation via a prismatic motor is needed, such as in high load carrying (using hydraulic devices). Therefore, we think the proposed solutions are of great interest to the scientific community.

\section{REFERENCES}

1 Lowen, G.G., Tepper, F.R. and Berkof, R.S. Balancing of linkages - an update. Mechanism and Machine Theory, 1983, 18(3), 213-230.

2 Arakelian, V. and Smith, M. Shaking force and shaking moment balancing of mechanisms: an historical review with new examples. Journal of Mechanical Design, 2005, 127(2), 334339 (see also ERRATUM. 2005, 127(5), 1034-1035). 
3 Arakelian, V., Dahan, M. and Smith, M.R. A historical review of the evolution of the theory on balancing of mechanisms. International Symposium on History of Machines and Mechanisms - Proceedings HMM2000, Kluwer Academic Publishers, Dordrecht/Boston/ London, 2000, pp. 291-300.

4 Agrawal, S.K. and Fattah, A. Reactionless space and ground robots: novel designs and concept studies. Mechanism and Machine Theory, 2004, 39(1), 25-40.

5 Wang, J. and Gosselin, C.M. Static balancing of spatial three-degree-of-freedom parallel mechanisms. Mechanism and Machine Theory, 1999, 34(3), 437-452.

6 Newman, W.S. and Hogan, N. The optimal control of balanced manipulators. Proc. winter annual meeting of the ASME, 1986, Anaheim, California.

7 Laliberté, T., Gosselin, C.M. and Jean, M. Static balancing of 3-DOF planar parallel mechanisms. IEEE/ASME Transactions on Mechatronics, 1999, 4(4), 363-377.

8 Fujikoshi, K. Balancing apparatus for jointed robot. Japanese Patent JP51-122254, 26 October 1976.

9 Wang, J. and Gosselin, C.M. Static balancing of spatial four-degree-of-freedom parallel mechanisms. Mechanism and Machine Theory, 2000, 35(4), 563-592.

10 Russo, A., Sinatra, R. and Xi, F. Static balancing of parallel robots. Mechanism and Machine Theory, 2005, 40(2), 191-202.

11 Ouyang, P. and Zhang, W.J. Force Balancing of Robotic Mechanisms based on Adjustment of Kinematic Parameters. ASME Journal of Mechanical Design, 2005, 127(3), 433-440.

12 Berkof, R.S. Complete fore and moment balancing of inline four-bar linkages. Mechanism and Machine Theory, 1973, 8(3), 397-410.

13 Arakelian, V. and Smith, M.R. Complete shaking force and shaking moment balancing of linkages. Mechanism and Machine Theory, 1999, 34(8), 1141-1153. 
14 Dresig, H., Naake, S. and Rockausen, L. Vollständiger und harmonischer Ausgleich ebener Mechanismen. VDI Verlag, Düsseldorf, 1994.

15 Herder, J.L. and Gosselin, C.M. A counter-rotary counterweight for light-weight dynamic balancing. Proc. ASME 2004 DETC/CIEC Conference, 28 September - 2 October 2004, Salt Lake City, Utah, USA, pp. 659-667.

16 Kochev, I.S. General theory of complete shaking moment balancing of planar linkages: a critical review. Mechanism and Machine Theory, 2000, 35(11), 1501-1514.

17 Fattah, A. and Agrawal, S.K. On the design of reactionless 3-DOF planar parallel mechanisms. Mechanism and Machine Theory, 2006, 41(1), 70-82.

18 Ricard, R. and Gosselin, C.M. On the development of reactionless parallel manipulators. Proc. ASME 2000 DETC, 10-13 September 2000, Baltimore, Maryland, USA.

19 Wu, Y. and Gosselin, C.M. On the synthesis of a reactionless 6-DOF parallel mechanism using planar four-bar linkages. Proc. Workshop on Fundamental Issues and Future Research Directions for Parallel Mechanisms and Manipulators, 3-4 October 2002, Quebec City, Quebec, Canada.

20 Gosselin, C.M., Côté, G. and Wu, Y. Synthesis and design of reactionless three-degree-offreedom parallel mechanisms. IEEE Transaction on Robotics and Automation, 2004, 20(2), $191-199$.

21 Foucault, S. and Gosselin, C.M. Synthesis, design, and prototyping of a planar three degrees-of-freedom reactionless parallel mechanism. Journal of Mechanical Design, 2004, 126(6), 992-999.

$22 \mathrm{Wu}$, Y. and Gosselin, C.M. Design of reactionless 3-DOF and 6-DOF parallel manipulators using parallelepiped mechanisms. IEEE Transaction on Robotics and Automation, 2005, 21(5), 821-833. 
23 Papadopoulos, E. and Abu-Abed, A. Design and motion planning for a zero-reaction manipulator. Proc. IEEE International Conference on Robotics and Automation (ICRA), 1994, San Diego, CA, USA, pp. 1554-1559.

24 Arakelian, V. and Briot, S. Dynamic balancing of the SCARA robot. The 17th CISMIFToMM Symposium on Robot Design, Dynamics, and Control (RoManSy 2008), 5-9 July 2008, Tokyo, Japan.

25 Bagci, C. Complete shaking force and shaking moment balancing of link mechanisms using balancing idler loops. ASME Journal of Mechanical Design, 1982, 104, 482-493.

26 Frolov, K.V. Theory of mechanisms and machines. Moscow, ed. Vishaya shkola, 1987.

27 Doronin, V.I. and Pospelov, A.I. Balanced slider-crank mechanism", Patent SU1627769, 15 February 1991.

28 Hilpert, H. Weight balancing of precision mechanical instruments. Mechanisms, 1968, 3(4), 289-302.

29 Arakelian, V. Equilibrage dynamique complet des mécanismes. Mechanism and Machine Theory, 1998, 33(4), 425-436.

30 Leblond, M. and Gosselin, C.M. Static balancing of spatial and planar parallel manipulators with prismatic actuators. Proc. ASME 1998 DETC Conference, 1998, Atlanta, Georgia, USA, pp. 1-12.

31 Baradat, C., Arakelian, V., Briot, S. and Guegan, S. Design and prototyping of a new balancing mechanism for spatial parallel manipulators. ASME Journal of Mechanical Design, 2008, (to be published).

32 Seyferth, W. Massenersatz duch punktmassen in räumlichen getrieben. Mechanism and Machine Theory, 1974, 9(1), 49-59. 
$33 \mathrm{Wu}$, Y. and Gosselin, C.M. On the dynamic balancing of multi-DOF parallel mechanisms with multiple legs. Transaction of the ASME. Journal of Mechanical Design, 2007, 129(2), 234-238. 


\section{List of figures}

Figure 1. Complete shaking force and shaking moment balanced 3-RRR planar parallel manipulators.

Figure 2. Schematic of the 3-RPR robot under study.

Figure 3. Schematics of the $3-R P R$ mechanism with the added $R R R$ chain used for the cancellation of the shaking force and shaking moment.

Figure 4. Shaking force and shaking moment before (solid line) and after (dashed line) the addition of the counterweights, and after the addition of the counter-rotations (gray line).

Figure 5. A general slider-crank mechanism.

Figure 6. A manipulator leg with added Scott-Russell mechanism.

Figure 7. Schematics of a shaking force and shaking moment balanced 3-RPR mechanism.

Figure 8. Shaking force and shaking moment, before (solid line) and after (dashed line) the addition of the counterweights, and after the addition of the counter-rotations (gray line).

Figure 9. Complete shaking force and shaking moment balancing of planar manipulators with prismatic pairs via structural modification of one leg.

Figure 10. Complete shaking force and shaking moment balancing of planar manipulators with prismatic with reduced number of Scott-Russell mechanisms. 